\title{
Miopia na Infância
}

\author{
Rosana Nogueira Pires da Cunha*
}

\section{Definições}

As miopias se dividem em primárias e secundárias. As formas primárias se subdividem em fisiológicas, intermediárias, patológicas e por curvatura. As formas secundárias decorrem de alterações estruturais no globo ocular, como exemplo as secundárias ao alongamento do globo, como no glaucoma juvenil.

Os vários tipos de miopia primária podem ser diferenciados pelo comprimento axial do globo ou, mais frequentemente, por seu estado refratométrico: aqueles com miopia abaixo de -3,00 dioptrias (D) são portadores de miopia fisiológica; os que apresentam entre $-3,00$ e $-5,00 \mathrm{D}$ apresentam tanto miopia fisiológica como intermediária e aqueles com refrações entre $-5,00$ e - $8,00 \mathrm{D}$ possuem miopia intermediária ou patológica. Indivíduos com miopia superior a $-8,00 \mathrm{D}$ são portadores, invariavelmente, de miopia patológica.

$\mathrm{Na}$ miopia fisiológica os olhos são normais quanto ao aspecto fundoscópico: na intermediária o comprimento axial está aumentado e as alterações fundoscópicas são mínimas, como a formação de crescentes peripapilares e discreta palidez da coróide. Na miopia patológica ocorre alongamento do globo e alterações em todas as camadas do olho. Ao exame fundoscópico podem se observar estafilomas posteriores, degeneração macular e membranas neovasculares.

Os indivíduos com miopia por curvatura são aqueles que apresentam alterações importantes na córnea, como ceratocone, ou no cristalino, como esferofacia. Como o comprimento axial do globo nesses casos é normal, o risco de degenerações na retina não difere dos olhos emétropes.

\section{Epidemiologia}

Os dados epidemiológicos referentes à prevalência de miopia são variados, devido às diferentes metodologias empregadas, aos diversos valores aceitos para consideração de miopia (-0.25 D a -1.00 D), aos erros conseqüentes de exames sem cicloplegia, e ainda, à não expressão dos valores em equivalentes esféricos.

* Setor de Estrabismo - Depto. de Oftalmologia - Universidade Federal de São Paulo - UNIFESP.
A prevalência da miopia varia nos diferentes grupos etários, sendo mais comum o início em crianças e adultos jovens ${ }^{1}$.

A prevalência da miopia também varia conforme a etnia. Em escolares nascidos em Hong Kong a prevalência chega a ser $62 \%$ na faixa etária de 6 a 17 anos. Nesse grupo a miopia começa usualmente a se manifestar aos 6 anos de idade e apresenta uma taxa de progressão maior do que a observada em crianças européias ${ }^{2}$. Estudos epidemiológicos feitos em escolares de Taiwan revelam prevalência de $56 \%$ de míopes aos 12 anos de idade e $84 \%$ aos 18 anos ${ }^{3}$. Na Índia a prevalência de miopia na população urbana com idade entre 11 e 20 anos é de $16,6 \%{ }^{4}$ enquanto nos EUA é de $25,7 \%$ entre 12 e 17 anos, sendo menor $(11,7 \%)$ na população negra ${ }^{5}$.

A prevalência e grau da miopia foram maiores em meninas em uma pesquisa feita em Taiwan ${ }^{3}$, podendo ter havido influência do sexo relacionada à etnia.

Finalmente fatores sócio-econômicos e culturais também podem influenciar a prevalência e progressão da miopia. Dados epidemiológicos mostram haver maior prevalência em áreas urbanas que em áreas rurais ${ }^{6} \mathrm{e}$ existem mais míopes em profissões que exigem trabalhos para perto envolvendo detalhes ${ }^{7}$.

\section{Etiologia}

Não existe uma única causa para a miopia, podendo a etiologia ser genética ou ambiental. Três fatores são importantes para o seu desenvolvimento:

3.1. Relação entre o esforço visual para perto e uma fraca acomodação.

3.2. Predisposição hereditária.

3.3. Relação entre a pressão intra-ocular e debilidade escleral.

Existe maior influência dos dois primeiros fatores, em graus diferentes, no período inicial da miopia, enquanto o terceiro fator é mais freqüente nos graus mais avançados, causando sua progressão ${ }^{8}$.

Em relação ao primeiro fator, o esforço visual resultante do trabalho para perto, somado à influência de distúrbios refrativos, acarretando desfocalização óptica, durante o período de crescimento do globo, faria com que o eixo ânteroposterior se tornasse mais longo. Tal tipo de miopia não costuma ultrapassar 3 graus ${ }^{9,10}$. 
Quanto ao segundo fator, de predisposição hereditária, estudos mostram que a herança pode ser autossômica dominante, recessiva e poligênica. Na miopia autossômica dominante a miopia se desenvolve tardiamente na infância e usualmente não atinge altos graus. A miopia autossômica recessiva é característica de comunidades com alta freqüência de consanguinidade, mas também está relacionada a alguns casos esporádicos ${ }^{11}$. Analisando três gerações de uma amostra da população chinesa ${ }^{12}$, estabeleceram que o desenvolvimento da miopia segue um modelo poligênico e multifatorial, no qual a influência genética permanece constante, enquanto a influência ambiental mostrase aumentada nas três últimas gerações.

Na miopia patológica existe sempre uma história familiar, havendo evidências de ser decorrente de herança autossômica recessiva ${ }^{13}$.

No terceiro fator, a debilidade da esclera pode estar relacionada a fibrilogênese defeituosa, a qual pode ser congênita ou resultante de doenças sistêmicas. Existe uma resposta inadequada ao crescimento do globo e subseqüente alongamento, mesmo com a pressão intra-ocular normal. $\mathrm{O}$ alongamento se localiza principalmente na porção posterior do globo, produzindo alterações adversas da coróide e retina.

\section{Fatores de risco}

4.1. História familiar: é um fator de risco para o desenvolvimento da miopia, já citado no sub-título etiologia.

4.2. Prematuridade: o risco de ter um erro refrativo e não somente miopia, é maior em prematuros que em recém-nascidos a termo ${ }^{14}$. A incidência de miopia em recém-nascidos prematuros, com ou sem retinopatia da prematuridade (ROP) é de $8 \%$ aos 6 meses de idade, podendo ser transitória ${ }^{14,15}$.

Existem dúvidas se maior freqüência de miopia também ocorre em prematuros que não desenvolveram ROP. Para alguns autores, mesmo estes apresentam um risco maior de ter miopia e anisometropia ${ }^{14}$. Para outros ${ }^{16,17}$ não existe maior freqüência de miopia em prematuros ou recém-nascidos com baixo peso se não possuem ROP. De qualquer modo, é fato que nos casos com ROP moderada a severa, existe maior incidência de miopia ${ }^{17,18}$, variando entre 30 e $40 \%$ nos casos tratados com criocauterização ${ }^{14}$. As alterações mais significantes na refração ocorrem entre 3 e 12 meses de idade ${ }^{18}$.

O motivo pelo qual os prematuros apresentam miopia deve-se provavelmente a valores oculométricos: córnea mais curva e comprimento axial maior em relação aos recémnascidos a termo ${ }^{19}$.

O peso ao nascer, a severidade da ROP e a presença de miopia aos 3 meses de idade pressupõem alta miopia aos 5 anos ${ }^{18}$.

4.3. Refração estática: quando próxima a emetropia (menor que $+0,75 \mathrm{D})$ ou propriamente emetrópica, em crianças, pode indicar miopia em um futuro próximo ${ }^{20}$.

4.4. Esforço visual para perto: o uso de computadores e o hábito de assistir à televisão têm sido considerados fatores de risco para a miopiogênese ou para sua progressão em jovens e adultos, mas não há comprovação em relação a outras formas de trabalho para perto.

Provavelmente existem outros fatores, como alterações na acomodação induzidas pela fadiga ${ }^{21}$. A distância de leitura, envolvendo a postura da criança, assim como a iluminação do ambiente de estudo, também têm sido pesquisados como possívei s fatores desencadeantes da miopia ${ }^{22}$.

4.5. Esoforia: a presença de esoforia para perto parece ser também um fator de risco para o início de miopia em jovens ${ }^{23,24}$. Medindo-se a acomodação tônica em crianças de 7 a 16 anos, antes e após 15 minutos de jogo com vídeo ("video-game"), notou-se que ocorria aumento significante em míopes, o mesmo não ocorrendo em hipermétropes e emétropes ${ }^{9}$. Outra evidência seria o fato de pacientes com alta miopia e esotropia, quando submetidos a cirurgia tradicional de correção do estrabismo, apresentarem maior freqüência de esotropia residual e recurrente, sendo o estímulo constante para a convergência acomodativa aparentemente a causa ${ }^{25}$.

4.6. Pressão intra-ocular: pode ser maior em olhos míopes em relação aos não míopes, mas não está comprovado se existe influência no crescimento anormal do globo ${ }^{26}$. Crianças que se tornaram míopes, e foram examinadas alguns meses após, não apresentaram pressão intra-ocular aumentada ${ }^{27}$.

\section{Progressão}

Existe relação entre a idade em que foi feito o diagnóstico, o sexo e a refração inicial. A taxa média de progressão foi maior em crianças míopes foi diagnosticada em uma idade entre 5 e 7 anos que em crianças entre 11 e 15 anos em uma pesquisa feita nos EUA ${ }^{28}$. O mesmo estudo revelou que também existe uma influência maior na taxa de progressão da miopia na puberdade, pois entre 8 e 10 anos de idade, a progressão foi maior em meninas. Além disso, notou-se também que crianças com mais de uma dioptria de miopia ao primeiro exame, apresentavam uma progressão mais rápida do que naquelas com menos grau.

Outros fatores influenciam a taxa de progressão da miopia. Lin e cols ${ }^{3}$ estudaram 11.178 indivíduos entre 6 e 18 anos, analisando o comprimento axial do globo com biometria ultrassônica, curvatura corneal e refração estática com autorefrator, tendo chegado às seguintes conclusões:

a) Crescimento axial do globo: apresenta uma relação direta com a progressão da miopia.

b) Profundidade da câmara anterior: aprofunda-se com a idade, tendo relação com a severidade da miopia.

c) Espessura do cristalino: diminui dos 7 aos 13 anos e aumenta com a idade e severidade da miopia, após os 15 anos. Nos pacientes que desenvolvem miopia, ao invés do poder refrativo do cristalino manter-se coordenado com o crescimento axial do globo, como parte do processo de emetropização, ocorrem alterações em sua curvatura, levando ao aumento da miopia, aproximadamente aos 10 anos de idade ${ }^{29}$. 
Comparando o efeito do trabalho para perto na miopia juvenil, Goss \& Rainey ${ }^{30}$ concluíram haver diferença na progressão da miopia relacionada à época do ano, sendo que nos meses de férias escolares mais prolongadas (verão), a progressão da miopia era menor em relação aos meses de atividades escolares mais intensas.

Fatores relacionados com a resposta acomodativa e esoforia também têm sido associados com a progressão da miopia, como mencionado anteriormente em fatores de risco.

\section{Miopia congênita}

A miopia congênita apresenta características especiais. Existem três formas para explicar sua origem:

6.1. Miopia congênita devida à combinação de diâmetro antero-posterior longo e de alto poder refrativo (usualmente do cristalino). Quando a esclera é forte, normalmente esse tipo de miopia não progride: o alongamento do globo é compensado pela redução do poder refrativo do cristalino.

6.2. Miopia congenita decorrente de esclera fraca e em constante distensão: progride rapidamente e o prognóstico é pobre.

6.3. Miopia congênita associada a anomalias de desenvolvimento do globo ocular. Este tipo de miopia é freqüentemente complicado por outros tipos de defeitos, como colobomas, sub-luxação do cristalino, atrofia do nervo óptico, etc.

\section{Associações sistêmicas e oculares de miopia}

7.1. Sistêmicas: Síndrome de Down, Síndrome de Marfan, Homocistinúria, Síndrome de Ehlers-Danlos, Síndrome de Stickler, Síndrome de Pierre-Robin, Síndrome de PraderWilli, Síndrome de Noonan entre outras.

7.2. Oculares: catarata congênita, opacidades da córnea, ptose, distrofias da coróide e retina (retinose pigmentar, distrofia de cones, cegueira noturna congênita estacionária, coroideremia, atrofia "girata"), ectopia "lentis", persistência de fibras de mielina, retinopatia da prematuridade e albinismo.

\section{Quadro clínico}

8.1. Sinais e sintomas: o primeiro sinal de miopia é a redução da acuidade visual à distância, freqüentemente normalizada com lentes corretoras. As queixas mais típicas incluem fadiga ocular na visão de perto e borramento na visão de longe. A amplitude de acomodação está diminuída.

8.2 Alterações na musculatura ocular extrínseca (MOE): quando um paciente míope traz um objeto próximo aos seus olhos, os músculos retos mediais sofrem grande esforço, levando à fadiga muscular e sintomas de astenopia. Quando o esforço de convergência é insuficiente, a visão binocular é comprometida, resultando exoforia ou exotropia, que por sua vez reduzem mais o estímulo para convergir.

8.3. Alterações de fundo de olho: nos estágios iniciais da miopia não existem alterações importantes, exceto por alguns defeitos próximos ao nervo óptico. Na miopia congênita ou hereditária existem alterações, mas estas são mais características de miopia severa. Comumente a miopia permanece em grau baixo ou moderado, mas às vezes o globo ocular continua a se alongar, aumentando o grau de miopia. O ponto remoto fica mais perto do olho, a amplitude de acomodação diminui, a fraqueza do músculo ciliar aumenta e a hemodinâmica piora.

As alterações na retina decorrentes de miopia maior que $6 \mathrm{D}$ aumentam com o tamanho do globo e com a idade ${ }^{31}$.

A progressão da miopia pode acarretar alterações oculares irreversíveis e perda da visão. As lentes não corrigem suficientemente a visão, a adaptação ao escuro pode ficar prejudicada e perdas no campo visual (escotomas centrais ou paracentrais) podem ocorrer. O alongamento da porção posterior do globo afeta inicialmente a área do disco óptico. Defeitos em forma de cone se desenvolvem, abrangendo o disco óptico como um anel. Às vezes o disco fica mais largo e achatado, acinzentado. Na miopia severa progressiva são observados estafilomas no pólo posterior, que se caracterizam por uma linha arqueada com os vasos retinianos se curvando sobre ele. Com a crescente atrofia da coróide e retina o processo degenerativo se acentua, surgindo roturas na membrana de Bruch e no epitélio pigmentário, conhecidas como "lacquer cracks". Estas lesões podem ocorrer na adolescência, não afetam por si só a acuidade visual mas representam um sinal de mau prognóstico e podem estar associados com defeito de cores para azul-amarelo ${ }^{32}$. Degenerações focais na retina, hemorragias e membranas vasculares sub-retinianas, associadas com os "lacquer cracks" são as responsáveis pelo mau prognóstico visual.

Hemorragias na retina, particularmente na mácula, não são raras na miopia patológica e podem ser de dois tipos: hemorragias agudas, causando severa diminuição da visão mas com recuperação total, decorrentes de processo espontâneo ou manobra de Valsalva; hemorragias associadas a membranas neovasculares sub-retinianas, acarretando cicatrizações e manchas de Fuchs. Nestes o prognóstico é ruim pois as lesões podem ser seguidas de atrofia, irreversíveis na maioria dos casos ${ }^{33}$.

Áreas de atrofia de cório-retina, pequenas ou grandes podem ser observadas em ambos os olhos. Mesmo em olhos sem hemorragia macular podem ocorrer hiperpigmentação, a qual já é observada na infância ${ }^{1}$.

\section{Diagnóstico}

9.1. História: deve incluir questões sobre o início dos distúrbios visuais, seu tipo de atividade (nível escolar - crianças no "jardim da infância" não precisam ler à lousa ou detalhes para longe), história familiar de miopia.

9.2. Exame da MOE: deve incluir teste de cobertura, avaliação sensorial, convergência e avaliação da reserva acomodativa. Quando há suspeita de paresia ou espasmo de acomodação, deve-se fazer o exame sob cicloplegia.

9.3. Refração estática: se as lentes corretoras não produzi- 
rem acuidade visual normal, ambliopia ou lesões orgânicas devem ser pesquisadas.

9.4. Exame ocular dos meios ópticos e fundo, incluindo biomicroscopia do vitreo e retina, exame de oftalmoscopia binocular direta e indireta.

\section{Tratamento}

10.1. Prescrição das lentes: deve-se prescrever o mínimo de lentes esféricas e cilíndricas, que forneça a melhor visão. Anisometropia de 2 dioptrias ou mais é um indicador importante de correção com óculos, podendo ser indicado o uso de lentes de contato, apesar de a maioria das crianças tolerar bem óculos com altos graus de anisometropia.

10.2. Seguimento: exames anuais permitem julgar a progressão da miopia. Quando astigmatismo está presente, calcula-se o grau pelo equivalente esférico. Para estimar a taxa de progressão em um ano, usa-se a seguinte fórmula:

$$
\mathbf{G A}=\frac{\text { EE 2 - EE 1 }}{\mathbf{T}}
$$

Onde GA é o gradiente de progressão em um ano, EE2 significa o esférico equivalente ao final de um ano, EE1 o equivalente esférico no início do seguimento e $\mathbf{T}$ o tempo em anos. O gradiente de menos de uma dioptria em um ano indica miopia de progressão lenta, o de uma ou mais, progressão rápida ${ }^{8}$.

\section{REFERÊNCIAS BIBLIOGRÁFICAS}

1. Curtin BJ. The myopias: Basic Science and Clinical Management. Philadelphia, Harper \& Row Publishers, 1985.

2. Lam CS, Edwards M, Millodot M, Goh WS. A 2-year longitudinal study of myopia progression and optical component changes among Hong Kong school children. Optom Vis Sci 1999;76(6):370-80.

3. Lin LL, Shih YF, Tsai CB, Chen CJ, Lee LA, Hung PT, Hou PK. Epidemiologic study of ocular refraction among schoolchildren in Taiwan in 1995. Optom Vis Sci 1999;76(5):275-81.

4. Mohan M, Pakrasi S, Zutshi R. Myopia in India. Acta Ophthalmol 1988; $185: 19$

5. Sperduto RD, Seigel D, Roberts J, Rowland M. Prevalence of myopia in the United States. Arch Ophthalmol 1983;101:405-7

6. Lithander J. Prevalence of myopia in school children in the Sultanate of Oman: a nation-wide study of 6292 randomly selected children. Acta Ophthalmol Scand 1999;77(3):306-9.

7. Fong DS, Pruett RC. Systemic associations with myopias. In Albert DM \& Jakobieck FA, eds. Principles and practice of Ophthalmology: clinical practice. Philadelphia: WB Saunders, 1994; cap.255.

8. Avetisov E. Myopia in children. In Taylor D, ed. Pediatric Ophthalmology. London: Blackwell Scientific Publication, 1990; cap 6.

9. Gwiazda J, Bauer J, Thorn F, Held R. Shifts in tonic accomodation after near work are related to refractive errors in children. Ophthalmic Physiol Opt 1995;15(2):93-7.

10. Goss DA, Rainey BB. Relationship of accomodative response and nearpoint phoria ia a sample of myopic children. Optom Vis Sci 1999;76(5):292-4.

11. Pacella R, McLellan J, Grice K, Del Bono EA, Wiggs JL, Gwiazda JE. Role of genetics factors in the etiology of juvenile-onset myopia based on a longitudinal study of refractive error. Optom Vis Sci 1999;76(6):381-6.

12. Wu MM, Edwards MH. The effect of having myopic parents: an analysis of myopia in three generations. Optom Vis Sci 1999;76(6):387-92.

13. Mantyjarvi M. High myopia in a family. Proceedings of the Fourth International Conference on myopia, Myopia International Research Foundation, Inc., 1990.

14. Holmström M, el Azazi M, Kugelberg U. Ophthalmological long-term followup of preterm infants: a population based, prospective study of the refraction and its development. Br J Ophthalmol 1998;82:1265-71.

15. Fledelius HC. Myopia of prematurity, clinical patterns. A follow-up of Danish children now aged 3-9 years. Acta Ophthalmol Scand 1995;73(5):402-6.

16. Saw SM, Chew SJ. Myopia in children born premature or with low birth weight. Acta Ophthalmol Scand 1997;75(5):548-50.

17. Pennefather PM, Tin W, Strong NP, Clarke MP, Dutton J, Cotrell DG. Refractive errors in children born before 32 weeks gestation. Eye 1997;11:736-43.

18. Quinn GE, Dobson V, Kivlin J, Kaufman LM, Repka MX, Reynolds JD, Gordon RA, Hardy RJ, Tung B, Stone RA. Prevalence of myopia between 3 months and $51 / 2$ years in preterm infants withand without retinopathy of prematurity. Cryoterapy for Retinopathy of Prematurity Cooperative Group. Ophthalmology 1998;105(7):1292-300.

19. Fledelius HC. Pre-term delivery and subsequent ocular development. Acta Ophthalmol Scand 1996;74(3):301-5.

20. Zadnik K, Mutti DO, Friedman NE, Qualley PA, Jones LA, Qui P, Kim HS, Hsu JC, Moeschberger ML. Ocular predictors of the onset of juvenile myopia. Invest Ophthalmol Vis Sci 1999; 40(9):1936-43.

21. Mutti DO, Zadnik K. Is computer use a risk factor for myopia? J Am Optom Assoc 1996;67(9):521-30.

22. Saw SM, Nieto FJ, Katz J, Chew SJ. Distance, lighting and parental beliefs: understanding near work in epidemiologic studies of myopia. Optom Vis Sci 1999;76(6):355-62.

23. Drobe B, de Saint André R. The pre-myopic syndrome. Ophthalmic Physiol Opt 1995;15(5):375-8.

24. Goss DA, Jackson TW. Clinical findings before the onset of myopia in youth: 3. Heterophoria. Optom Vis Sci 1996;73(4):269-78.

25. Shauly Y, Miller B, Meyer E. Clinical characteristics and long-term postoperative results of infantile esotropia and myopia. J Pediatr Ophthalmol Strabismus 1997;34(6):357-64.

26. Goss DA, Caffey TW. Cinical findings before the onset of myopia in youth: 5 . Intraocular pressure. Optom Vis Sci 1999;76(5):286-91.

27. Quinn GE, Berlin JÁ, Young TL, Ziylan S, Stone RA. Ophthalmology 1995;102(2):180-5.

28. Braun CI, Freidlin V, Sperduto RD, Milton RC, Strahlman ER. The progression of myopia in school age children: data from the Columbia Medical Plan. Ophthalmic Epidemiol 1996;3(1):13-21.

29. Mutti DO, Zadnik K, Fusaro RE, Friedman NE, Sholtz RI, Adams AJ. Optical and structural development of the crystalline lens in childhood. Invest Ophthalmol Vis Sci 1998;39(1):120-33.

30. Goss DA, Rainey BB. Relation of childhood myopia progression rates to time of year. J Am Optom Assoc 1998;69(4):262-6.

31. Gözüm N, Cakir M, Gücukoglu A, Sezen F. Eur J Ophthalmol 1997;7(3):277-82.

32. Klein RM, Curtin BJ. Lacquer crack lesions in pathologic myopia. Am J Ophthalmol 1981;91:177-83.

33. Tokoro T, Mutamatsu T. Macular hemorrhage associated with high myopia. Proceedings of the Fourth International Conference on Myopia. Myopia International Research Foundation, Inc., 1990.

\section{Novidades na Internet!!!}

Agora no site CBO você tem disponível todas as informações na íntegra dos Arquivos Brasileiros de Oftalmologia http://www.cbo.com.br/abo 